\title{
The Predominant Neural Stem Cell Isolated from Postnatal and Adult Forebrain But Not Early Embryonic Forebrain Expresses GFAP
}

\author{
Tetsuya Imura, ${ }^{1}$ Harley I. Kornblum, ${ }^{2,3}$ and Michael V. Sofroniew ${ }^{1}$ \\ Departments of ${ }^{1}$ Neurobiology, ${ }^{2}$ Pharmacology, and ${ }^{3}$ Pediatrics, and Brain Research Institute, University of California, Los Angeles, California $90095-1763$
}

Periventricular germinal zones (GZs) of developing and adult brain contain neural stem cells (NSCs), the cellular identities and origins of which are not defined completely. We used tissue culture techniques and transgenic mice expressing herpes simplex virus thymidine kinase (HSV-TK) from the mouse glial fibrillary acid protein (GFAP) promoter to test the hypothesis that certain NSCs express GFAP. To do so, we determined the relative proportions of multipotent neurospheres that are formed by GFAP-expressing cells derived from GZs at different stages of development. In this transgenic model, dividing GFAP-expressing cells are ablated selectively by treatment with the antiviral agent ganciclovir (GCV). Single-cell analysis showed that transgene-derived HSV-TK was present only in GFAP-expressing cells. GCV applied in vitro eliminated growth of multipotent neurospheres from GZs of postnatal and adult transgenic mice but not early embryonic (embryonic day 12.5) transgenic mice. GCV prevented growth of secondary multipotent neurospheres prepared after passage of primary transgenic neurospheres derived from all three of these developmental stages. In addition, GCV prevented growth of multipotent neurospheres from transgenic astrocyte-enriched cell cultures derived from postnatal GZ, and elaidic acid GCV given for $4 \mathrm{~d}$ to adult transgenic mice in vivo abolished the ability to grow multipotent neurospheres from GZ. Extensive control experiments, including clonal analysis, demonstrated that failure of neurosphere growth was not merely secondary to loss of GFAP-expressing support cells or the result of a nonspecific toxic effect. Our findings demonstrate that the predominant multipotent NSCs isolated from postnatal and adult but not early embryonic GZs express GFAP, and that NSCs exhibit heterogeneous expression of intermediate filaments during developmental maturation

Key words: neural stem cell; neural progenitor; neurogenesis; astrocyte; ventricular zone; brain; glial fibrillary acid protein

\section{Introduction}

Neural stem cells (NSCs) are multipotent, self-renewing progenitors that can give rise to neurons, oligodendrocytes, and astrocytes. NSCs are present in periventricular germinal zones (GZs) of both developing and adult brain from early embryonic stages until senescence (Gage, 2000; Anderson, 2001; Temple, 2001). The biology of NSCs is of considerable interest, reflecting not only their fundamental contribution to building the developing CNS but also their potential functional roles in the adult forebrain and their promise for treating neurological illness. Although they have been studied extensively, the identities of NSCs are not defined completely, and the relationship between NSCs in developing and adult brain is uncertain.

Cell lineage analyses in vivo with markers of cell division, electron microscopy, and retroviral markers suggest that at least some NSCs in the adult GZ express glial fibrillary acidic protein (GFAP) and exhibit ultrastructural characteristics of astroglia (Doetsch et al., 1999; Alvarez-Buylla et al., 2001). Consistent with

Received 0ct. 18, 2002; revised Jan. 13, 2003; accepted Jan. 14, 2003.

This work was supported by National Institutes of Health Grants MH065756 (H.I.K.) and NS42039 (M.V.S.) and fellowships from the Uehara Memorial Foundation and the Japan Heart Foundation (T.I.). We thank N. B. Doan and R. A. Lane for technical assistance and A. D. R. Garcia for confocal analysis of forebrain tissue and helpful discussion. We are grateful to P. Borgese and Hoffman La Roche for ganciclovir and to F. Myhren and ConPharma for E-GCV.

Correspondence should be addressed to Michael V. Sofroniew, Department of Neurobiology, University of California, Los Angeles School of Medicine, 10833 Le Conte Avenue, Los Angeles, CA 90095-1763. E-mail: sofroniew@mednet.ucla.edu.

Copyright $\odot 2003$ Society for Neuroscience $\quad 0270-6474 / 03 / 232824-09 \$ 15.00 / 0$ this proposal are findings that GFAP-expressing cells in primary astroglial tissue cultures of postnatal or adult GZs have NSC potential (Laywell et al., 2000). These findings raise interesting questions about the nature of NSCs. If GFAP-expressing cells are confirmed as true NSCs, do these cells represent a minor or major subpopulation of NSCs? Given that NSCs are present at early embryonic stages before detectable expression of GFAP, do NSCs exist in multiple forms or have different characteristics at different stages of life? Given the association of GFAP expression with differentiated astrocytes, what are the implications of GFAP expression in NSCs?

Neural progenitor cells with characteristics of NSCs can be isolated from embryonic, perinatal, and adult GZs and grown in vitro as neurospheres that have self-renewing and multipotent potential. The ability to form multipotent neurospheres is currently the best in vitro assay for the presence of putative NSCs (Reynolds and Weiss, 1992; Luskin, 1993; Davis and Temple, 1994; Morshead et al., 1994, 1998; Tropepe et al., 1999; Geschwind et al., 2001; Svendsen et al., 2001; Capela and Temple, 2002). Cell-sorting techniques have been used to examine molecular characteristics of putative adult NSCs identified as neurosphere-forming cells. In one study, a partially enriched adult NSC population was found not to express GFAP (Rietze et al., 2001). In another study, a portion of a partially enriched adult NSC population was found to express GFAP, but the authors also suggested the presence of GFAP-negative cells with NSC potential (Capela and Temple, 2002). Thus, although several lines of 
evidence support the idea that at least some postnatal and adult NSCs express GFAP, there are conflicting findings.

In the present study, we used a transgenically targeted cellablation strategy to test the hypothesis that certain NSCs express GFAP and determined the relative contribution of GFAP-expressing cells to multipotent neurosphere formation at different developmental stages. We demonstrate that GFAP-expressing cells are the predominant multipotent neural progenitors isolated from early postnatal and adult but not early embryonic GZs and provide evidence that NSCs exhibit heterogeneous expression of intermediate filament proteins during developmental maturation in vivo and in vitro.

\section{Materials and Methods}

Animals. GFAP-thymidine kinase (GFAP-TK) transgenic mice were generated previously with a $15 \mathrm{~kb}$ promoter cassette that contained the full sequence of the mouse GFAP gene (Bush et al., 1998, 1999). This cassette (clone 445) contains all introns, promoter regulatory elements, exons, and $2 \mathrm{~kb}$ of $3^{\prime}$ and $2.5 \mathrm{~kb}$ of $5^{\prime}$ flanking regions; expression of GFAP is prevented by removal of a small fragment of the first exon (Johnson et al., 1995). This large and well studied promoter cassette has been used in many laboratories to generate many different lines of mice in which transgene expression has been shown to overlap with endogenous GFAP expression. All experimental and control animals were obtained by mating heterozygous females with wild-type C57BL/6 males, so that all transgenic and nontransgenic mice were derived from the same breeding colony back-crossed onto a C57BL/6 background (Bush et al., 1998). Transgenic mice that constitutively express the reporter protein green fluorescent protein (GFP) in all cells (Okabe et al., 1997) were used as a source of identifiable cells that did not express GFAP-TK for cell mixing studies. For developmental studies, vaginal plug identification was defined as embryonic day 0.5 (E0.5) and day of birth as postnatal day 0 (P0). Mice were housed in a $12 \mathrm{hr}$ light/dark cycle in a specific pathogen-free facility with controlled temperature and humidity and allowed ad libitum access to food and water, and experiments were conducted according to protocols approved by the Chancellor's Animal Research Committee of the Office for Protection of Research Subjects at the University of California at Los Angeles.

Neurospheres. Neurosphere cultures were prepared as described previously (Geschwind et al., 2001). E12.5, E15.5, or P1 cortices including periventricular zones were dissected, and meninges were removed. For adult ( $>2$ months) cultures, the lateral walls of the lateral ventricles were dissected and incubated with activated papain $(10 \mathrm{U} / \mathrm{ml})$ and DNase $(0.01 \%)$ at $37^{\circ} \mathrm{C}$ for $30 \mathrm{~min}$ followed by resuspension in ovomucoid inhibitor $(0.7 \mathrm{mg} / \mathrm{ml})$. Cells were dissociated by light trituration with a fire-polished glass pipette and resuspended at either 40,000 cells (for $\mathrm{E} 12.5$, E15.5, or P1) or 10,000 cells (for adult cells) per milliliter in DMEM/F12 medium (Mediatech, Herndon, VA), supplemented with B-27 (Invitrogen, San Diego, CA) and 20 $\mathrm{ng} / \mathrm{ml}$ fibroblast growth factor-2 (FGF2) (R\&D Systems, Minneapolis, MN). Epidermal growth factor (20 ng/ml; Sigma, St. Louis, MO) was added to adult cultures. Growth factors were added every $3 \mathrm{~d}$. To assess differentiation potential, $12 \mathrm{~d}$ in vitro (DIV) spheres were plated onto poly-L-lysine (PLL)-coated coverslips in neurobasal medium (Invitrogen) supplemented with B-27 in the absence of added growth factors for $5 \mathrm{~d}$. For quantitative analysis, 12 DIV spheres were plated onto PLL-coated coverslips and counted (a minimum of three coverslips per condition) with image-analysis software (MicroBrightField Inc., Williston, VT). Secondary neurosphere cultures were prepared by mechanical dissociation of 12 DIV primary neurospheres into single cells, passing of these cells through a $40 \mu \mathrm{m}$ nylon mesh, and resuspension at 10,000 live cells $/ \mathrm{ml}$.

Astrocyte-enriched cultures. Primary astrocyte cultures were prepared as described previously (Bush et al., 1998). P1 mouse cortex was dissected, and cells were dissociated by mechanical titration and seeded in T25 tissue culture flasks in DMEM/F12 with 10\% FBS at a density of $50,000 \mathrm{cells} / \mathrm{cm}^{2}$. Once confluence was attained (10-12 DIV), cells were shaken at $200 \mathrm{rpm}$ overnight to remove nonadherent cells. Remaining cells were trypsinized and replated (passage 1); when confluence was attained again, this procedure was repeated (passage 2). Most experiments were performed at passage 2 .

Neurospheres generated from astrocyte cultures. After passage, astrocytes were resuspended at a density of $40,000 \mathrm{cells} / \mathrm{ml}$ in neurosphere growth medium (DMEM/F12 with B-27 and $20 \mathrm{ng} / \mathrm{ml} \mathrm{FGF2} \mathrm{but} \mathrm{without}$ FBS). Cells formed floating spheres. FGF2 was added every $3 \mathrm{~d}$. After 14 DIV, spheres were plated on PLL-coated coverslips and either counted or differentiated by the withdrawal of FGF2 for $5 \mathrm{~d}$.

Clonal cultures. Methods for clonal cultures were adapted from previous studies (Tropepe et al., 1999; Geschwind et al., 2001; Groszer et al., 2001). Trypsinized, free-floating astroglia were passed through a $40 \mu \mathrm{m}$ nylon mesh, washed, and resuspended at a final total density of 1000 live cells per milliliter in neurosphere growth medium supplemented with filtered mouse neurosphere-conditioned medium.

Ganciclovir and elaidic acid ganciclovir. Ganciclovir (GCV), kindly donated by Hoffman La Roche (Nutley, NJ), was used in tissue cultures at a final concentration of $3 \mu \mathrm{m}$. Elaidic acid GCV (E-GCV), a potent lipophilic ester of ganciclovir (Balzarini et al., 1998) kindly donated by ConPharma (Oslo, Norway), was given to adult mice as single daily intraperitoneal injections at a dose of $100 \mathrm{mg} \cdot \mathrm{kg}^{-1} \cdot \mathrm{d}^{-1}$.

Immunocytochemistry. Cells plated on glass coverslips precoated with PLL (Sigma) were fixed in 4\% paraformaldehyde for $30 \mathrm{~min}$ and stained by immunofluorescence with the following primary antibodies: antiTuj1 (1:1000; Berkeley Antibodies, Richmond, CA), anti-GFAP (1:2000; Dako, Carpinteria, CA), anti-O4 (1:100; Chemicon, Temecula, CA), anti-nestin (Rat401; 1:100; Developmental Studies Hybridoma Bank,
A

E12.5

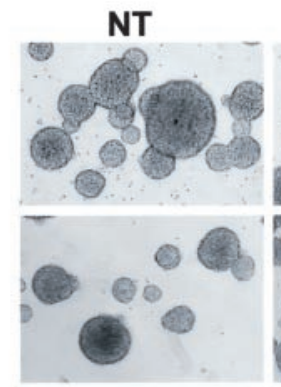

B

surivey

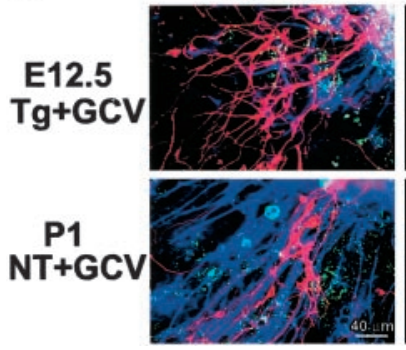

Tg

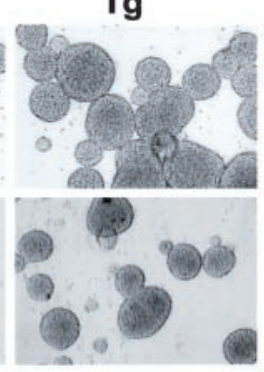

Truㅐ
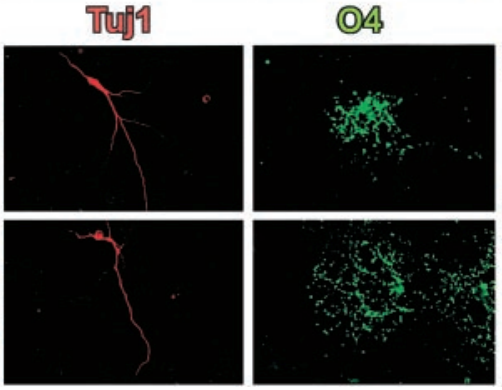

$\mathrm{Tg}+\mathrm{GCV}$

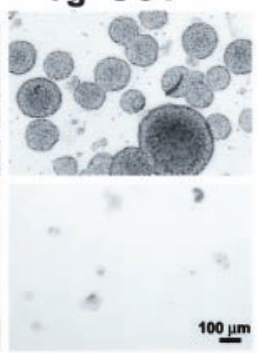

$100 \mu \mathrm{m}$

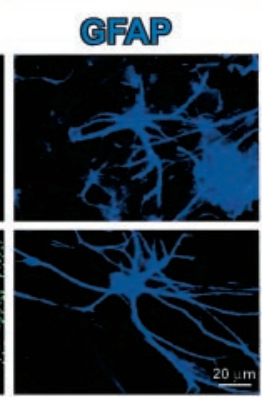

Figure 1. GFAP-expressing cells are required to form multipotent neurospheres from early postnatal but not early embryonic GZs. A, Phase-contrast images of live, floating neurospheres prepared from E12.5 or P1 tissue from nontransgenic (NT) or GFAP-TK transgenic $(T g)$ mice in the presence or absence of GCV. GCV completely prevented sphere growth from P1 transgenic mice but did not reduce growth from transgenic E12.5 or nontransgenic mice. $B$, Tricolored immunofluorescence of markers for neurons (Tuj1, red), oligodendrocytes ( 04, green) and astrocytes (GFAP, blue) after differentiation of neurospheres that were grown from E12.5 transgenic mice or P1 nontransgenic mice in the presence of GCV. Differentiation was induced in the absence of GCV. Photomicrographs show low-magnification surveys tripled labeled for all three markers, as well as details of each individual cell type. 
A primary neurosphere
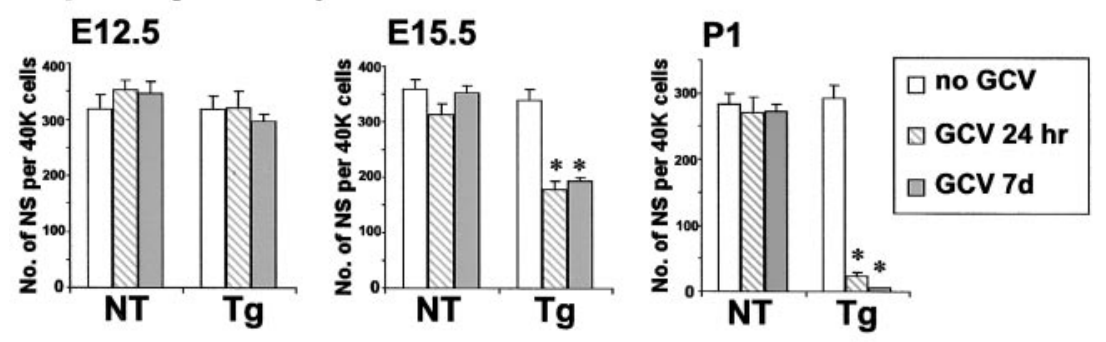

\section{B secondary neurosphere}

E12.5

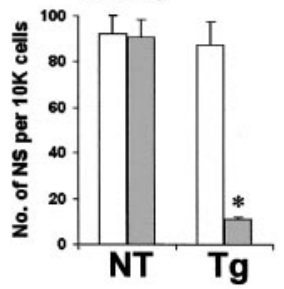

E15.5

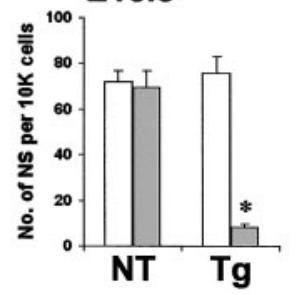

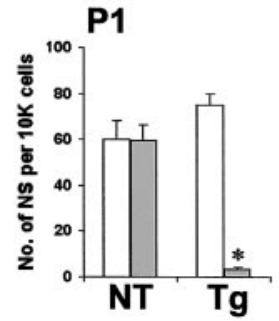

Figure 2. Quantitative analysis of multipotent neurosphere formation at different stages of development, with and without ablation of GFAP-expressing cells. Graphs show mean \pm SEM number of neurospheres (NS) prepared from E12.5, E15.5, or P1 tissue from nontransgenic (NT) or GFAP-TK transgenic $(T g)$ mice in the presence or absence of either $24 \mathrm{hr}$ or $7 \mathrm{~d}$ of GCV. A, Effects of GCV on the number of primary spheres formed per 40,000 cells derived directly from GZs. GCV treatment had no significant effect on the number of primary spheres derived directly from nontransgenic GZs at any age. GCV also had no significant effect on the number of primary spheres from E12.5 GZs but significantly reduced sphere formation by one-half at E15.5 and abolished sphere formation at P1. B, Effects of GCV on the number of secondary spheres formed per 10,000 cells derived after passage of primary spheres. GCV essentially abolished ( $>90 \%$ reduction) the formation of secondary spheres derived after passage of primary spheres from GZ at all three developmental stages. $n=3-8$ separate cultures prepared from different mice. *Significantly different from nontransgenic or non-GCV-treated mice ( $p<0.001$; ANOVA plus post hoc pairwise analysis).

The University of Iowa, Iowa City, IA), and anti-herpes simplex virus (HSV)-TK (1:1000) (Bush et al., 1998). Primary antibodies were visualized with Alexa 568- (red), Alexa 488- (green), and Alexa 350 (blue)conjugated secondary antibodies (Molecular Probes, Eugene, OR). Hoechst 33342 (blue) was used as a fluorescent nuclear counterstain. Stained cultures were examined and photographed by fluorescence microscopy (Zeiss, Oberkochen, Germany). For quantitative analysis, double- or single-labeled cells were counted (a minimum of three coverslips per condition) with image-analysis software (MicroBrightField Inc.). Tissue sections of $4 \%$ paraformaldehyde-fixed brains from adult mice were double stained (Bush et al., 1999) with primary antibodies: anti-HSV-TK (1:1000; P. Collins) visualized with an Alexa 568 (red)conjugated secondary antibody (Molecular Probes), combined with anti-GFAP (Dako) that was conjugated directly with Alexa 488 (green; Molecular Probes). Stained sections were examined and photographed by scanning confocal laser microscopy (Leica, Nussloch, Germany).

\section{Results}

To determine the relative contribution of GFAP-expressing cells to the neurogenic potential of embryonic, early postnatal, and adult GZ tissue, we used a combination of tissue culture techniques and a well characterized transgenic model in which HSV-TK is expressed from the mouse GFAP promoter with a promoter cassette comprising the entire mouse GFAP gene (Bush et al., 1998). Previous studies have demonstrated the precise and selective targeting of HSV-TK expression to GFAP-expressing cells by double-labeling immunocytochemistry at the single-cell level (Bush et al., 1999). Additional characterization of the overlap of GFAP and HSV-TK expression specifically in cells studied here is described below. In these GFAP-TK transgenic mice, the antiviral drug GCV kills dividing, GFAP-expressing cells both in vitro and in vivo (Bush et al., 1998, 1999). $\square$ no GCV

$\square$ GCV 7d
Ablation of GFAP-expressing cells abolishes the ability to derive NSCs from postnatal but not early embryonic GZs

We first determined the relative contribution of GFAP-expressing cells to NSCs isolated from GZ tissue at three developmental stages: early embryonic (E12.5), midembryonic (E15.5), and early postnatal (P1). NSCs were isolated and cultured as FGF2-responsive colony-forming, floating neurospheres prepared from GZ tissue of either nontransgenic or GFAP-TK transgenic mice. In the absence of GCV, the number of neurospheres generated (Figs. $1 A, 2 A$ ) and the ability of these spheres to differentiate into all three types of neural cells (Fig. $1 B$ ) did not differ between nontransgenic and transgenic mice for any age group. GCV added for the first 7 DIV had no detectable effect on sphere formation from nontransgenic mice of any age group (Figs. 1A, 2A) and did not alter the ability of nontransgenic spheres to differentiate into all three types of neural cells (Fig. 1B). GCV for 7 DIV also caused no detectable reduction in neurosphere formation from E12.5 tissue derived from transgenic mice (Figs. $1 A$, $2 A)$. In contrast, GCV for 7 DIV reduced neurosphere formation by approximately one-half from E15.5 tissue (Fig. 2A) and completely $(\geq 98 \%)$ prevented neurosphere formation from P1 GZ tissue derived from transgenic mice (Figs. $1 \mathrm{~A}, 2 \mathrm{~A}$ ). GCV was also effective in preventing sphere formation when added only during the first $24 \mathrm{hr}$ in neurosphere cultures (Fig. 2A), which suggests that GCV was ablating GFAPexpressing and dividing cells present at the onset of sphere formation rather than merely killing GFAP-expressing progeny derived from non-GFAP-expressing cells later during the culture period. GCV added after differentiation of transgenic neurospheres had no detectable effect on neurons or oligodendrocytes, which confirms the selective toxicity of GCV for dividing, GFAPexpressing cells. The observation that GCV had no effect on neurospheres grown from E12.5 tissue indicates that the neurosphere culture conditions do not select for GFAP-expressing NSCs or induce GFAP expression in all NSCs. Finally, it is of particular interest that GCV also prevented the formation of secondary spheres after passage of 12 DIV primary neurospheres derived from transgenic mice at all developmental stages (E12.5, E15.5, and P1), thus eliminating the capacity for self-renewal of neurospheres regardless of the age of the tissue used to form primary spheres (Fig. 2 B).

These results show that the potential of postnatal but not early embryonic GZ tissue to form neurogenic spheres in vitro was abolished by transgenically targeted ablation of dividing GFAPexpressing cells. This finding suggests that the predominant NSC in the postnatal GZ is a GFAP-expressing cell, which thus may be a type of astrocyte or related glia. In addition, the results are compatible with a model in which NSCs are heterogeneous with respect to GFAP expression and other molecules during embryogenesis (Suslov et al., 2002) either because there are multiple lineages of NSCs or because a single lineage gradually adopts 
GFAP expression. Alternative explanations are investigated below.

\section{Ablation of GFAP-expressing cells abolishes the ability to derive NSCs from primary cultures of astroglia}

To further study and characterize the NSC potential of GFAPexpressing glia, we used tissue cultures prepared as primary astrocytes derived from early postnatal GZ. Primary cell cultures enriched for astrocytes are reported to give rise to multipotent neurospheres when transferred to neurogenic conditions in vitro (Laywell et al., 2000). Because primary astrocyte cultures routinely contain at least 5\% GFAP-negative cells, it is possible that GFAP-negative NSCs could survive in such cultures and later proliferate to generate neurospheres and could in fact be the predominant sphere-forming cells. We therefore first determined the relative contribution of GFAP-expressing cells to neurosphere formation from primary astrocytes prepared from P1 mouse cortex by comparing the effects of GCV on cultures derived from nontransgenic and GFAP-TK transgenic mice. At 21 DIV, after one passage, primary astrocyte cultures formed confluent monolayers in which $\sim 95 \%$ of cells were GFAP-positive, and no Tuj1-positive neurons were detected (Fig. 3A,B). Immunocytochemistry and single-cell analysis showed that $>80 \%$ of GFAP-positive astroglia in cultures from transgenic mice were also immunoreactive for TK, and that all TK-positive cells also expressed GFAP; no TK-expressing cells were found to express markers of neurons or oligodendrocytes, and no cells were observed that were TK-positive and GFAP-negative (Fig. 4). The presence of some cells that are GFAP-positive and TK-negative is likely attributable to discontinuous expression of the GFAP gene in quiescent cells, combined with a longer intracellular half-life of the GFAP protein compared with the TK protein (Mucke et al., 1991).

Confirming a previous report (Laywell et al., 2000), we found that when cells cultured under primary astrocyte culture conditions (i.e., with serum) were transferred immediately after the second passage into neurosphere growth conditions (i.e., without serum and with FGF2), many floating cell aggregates formed that expressed nestin and were indistinguishable from neurospheres derived directly from $\mathrm{P} 1$ cortex (Fig. $3 C, D$ ). When these floating spheres were allowed to differentiate by withdrawal of FGF2 and plating after $14 \mathrm{DIV}$, they generated neurons, oligodendrocytes, and astrocytes, which demonstrates their multipotent NSC potential (Fig. 3E-G). GCV treatment during the first 7 DIV of neurosphere growth conditions completely (>98\%) abolished neurosphere formation from GFAP-TK transgenic astroglia, whereas sphere formation from nontransgenic astroglia was not affected (Fig. 3H,I). Neurospheres derived from these P1-derived primary astrocyte cultures also exhibited the capacity for selfrenewal by formation of secondary spheres after passage, and GCV prevented formation of secondary spheres, thus eliminating the capacity for self-renewal.

Together, these findings show that GFAP-expressing cells are necessary for neurosphere formation from primary astrocyte cultures, and they are compatible with a model in which the predominant NSC isolated from postnatal GZ is a type of GFAPexpressing astrocyte or related glia. Nevertheless, the data are also compatible with the possibilities that GFAP-expressing cells might provide essential support for the initial survival and proliferation of NSCs or that the GCV-induced death of GFAPexpressing cells might be toxic to NSCs.
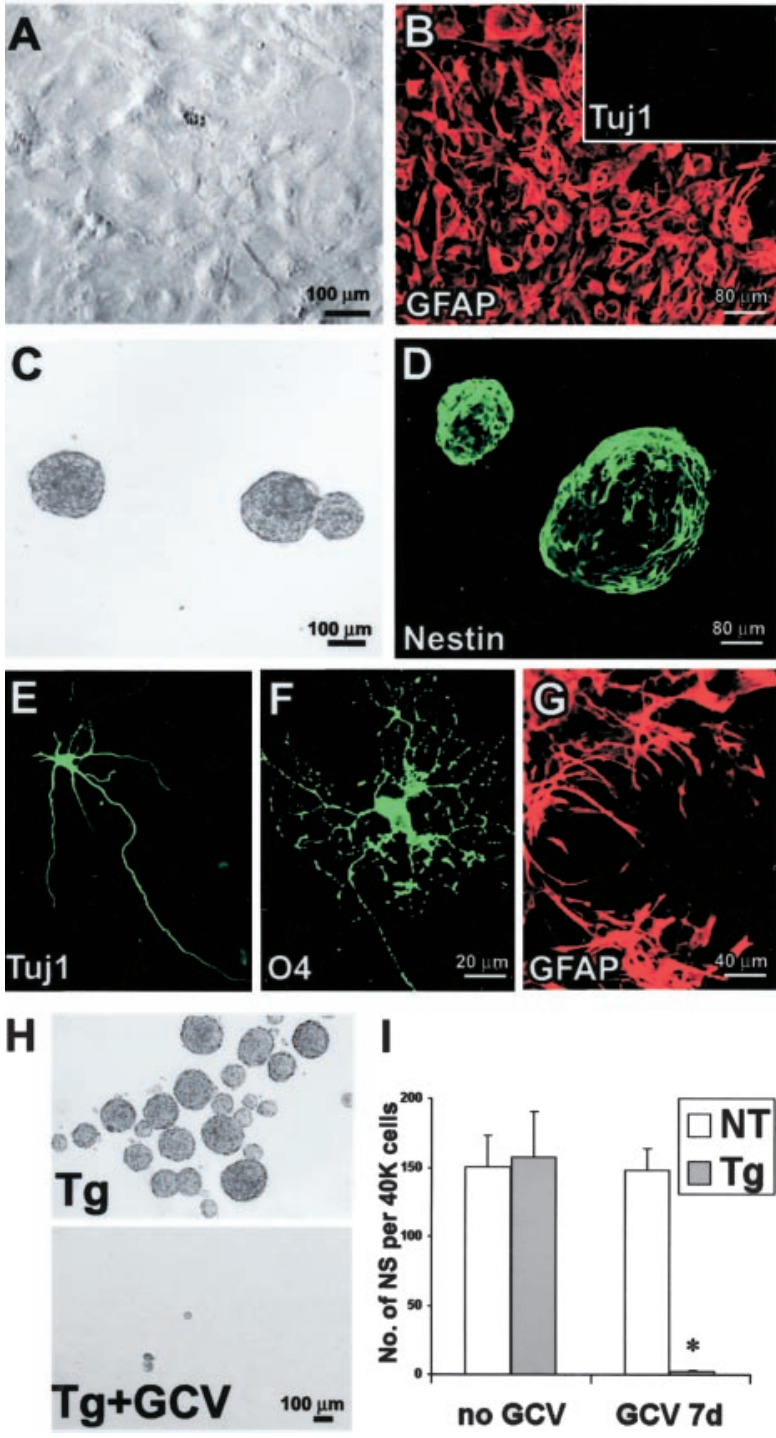

Figure 3. GFAP-expressing cells are required to form multipotent neurospheres from primary astrocyte cultures prepared from P1 GZs. A, Phase-contrast image of primary astrocytes in vitro showing high density of cells that have reached confluence after 21 DIV. $B$, Immunofluorescence of primary astrocyte culture at cell confluence after 21 DIV. Most cells stain for GFAP (red). No cells stain for Tuj1 (inset). C, Live floating neurospheres prepared from primary astrocyte culture. $D$, Fixed floating neurospheres prepared from primary astrocyte culture and stained for nestin (green). E-G, Immunofluorescence of chemical markers for neurons (Tuj1,E), oligodendrocytes $(04, F)$, and astrocytes (GFAP, G) shows cells of all three types derived by differentiation of neurospheres prepared from primary astrocyte cultures. $H$, Phase-contrast images of live floating neurospheres prepared from primary astrocyte cultures from GFAP-TK transgenic (Tg) mice in the absence or presence of GCV. I, Graph shows mean \pm SEM number of neurospheres (NS) formed per 40,000 cells prepared after passage of primary astrocyte cultures from nontransgenic (NT) or transgenic mice in the presence or absence of GCV. GCV completely prevented the growth of spheres from transgenic mice. $n=3-5$ separate cultures prepared from different mice. *Significantly different from nontransgenic or non-GCV-treated mice $(p<$ 0.001 ; ANOVA plus post hoc pairwise analysis).

\section{GFAP-expressing glia cultured from GZs are multipotent NSCs}

Our next goal was to differentiate between the following possibilities: (1) that GFAP-expressing cells have direct NSC potential (i.e., are both required and sufficient for multipotent neurogenesis), (2) that GFAP-expressing cells are merely support cells required for the formation of neurospheres, or (3) that ablation of differentiated astroglia is nonspecifically toxic to neurosphere 

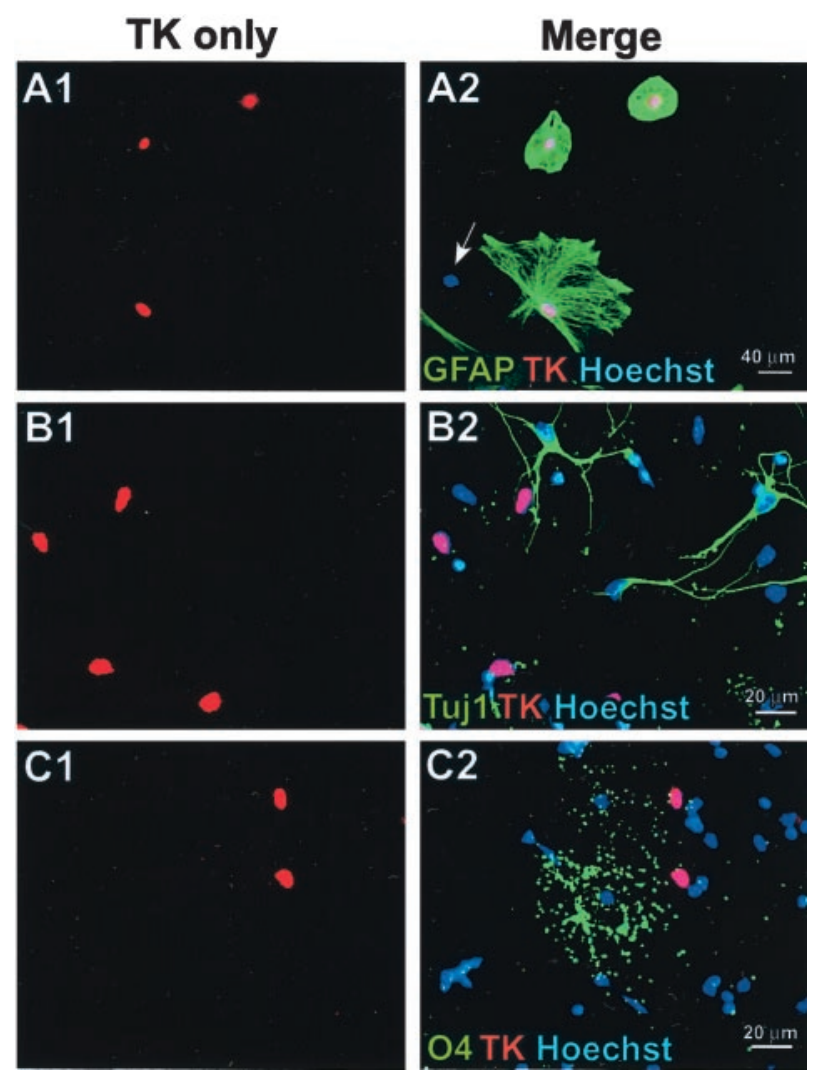

Figure 4. Transgene-derived HSV-TK is expressed only in GFAP-expressing cells in vitro.A-C, Primary astrocyte cultures derived from P1 GZs of GFAP-TK transgenic mice were double stained by immunofluorescence for TK (red) and either GFAP (A), Tuj1 (B), or 04 (C) (green); Hoechst blue was used as a general cytological counterstain. Pairs of images show the same microscopic fields with single-channel visualization of TK only (red) and merged visualization of all three fluorescent channels (red, green, blue). In merged images, red TK staining overlaps completely with blue stain, so that nuclei appear purple. A1, A2, All TK cells (red) are GFAP-positive (green) and vice versa. A cell negative for both GFAP and TK is indicated by the blue nucleus (arrow). B1, B2, No TK-positive cells (red) are positive for the neuronal marker Tuj1 (green). C1, C2, № TK-positive cells (red) are positive for the oligodendrocyte marker 04 (green).

formation. To do so, we used several approaches, including clonal analysis combined with mixing of transgenic cells derived from mice that expressed either GFAP-TK or GFP, analysis of chimeric GFAP-TK/GFP spheres, and experiments applying a 24 hr GCV pulse to confluent, nondividing GFAP-TK transgenic astroglia before passage.

Clonal analysis was performed in combination with transgenically targeted ablation of GFAP-expressing cells. Primary astrocyte cultures were prepared with cells derived either from mice that expressed GFAP-TK or mice that expressed GFP from a ubiquitous promoter active in all cells (Okabe et al., 1997). After passage, primary astrocyte cultures were dissociated and diluted until they consisted of suspensions of single cells. Mixed neurosphere cultures were then prepared from equal numbers of GFP and GFAP-TK astroglia at a final total density of 1000 cells $/ \mathrm{ml}$ (Fig. 3A). Suspensions of this cell density yield a high proportion (at least 95\%) of clonal, single-cell-derived neurospheres (Groszer et al., 2001). In current experiments with this starting cell density, mixed cultures of GFP and nontransgenic astrocytes suspended at equal numbers yielded either spheres that were entirely GFP positive or entirely GFAP-TK positive (Fig. $5 A$ ), and $<5 \%$ chimeric (green and white) spheres were observed, which indicates that the vast majority of spheres were derived clonally. In response to plating on adherent substrate and differentiation,

\section{A. clonal density}

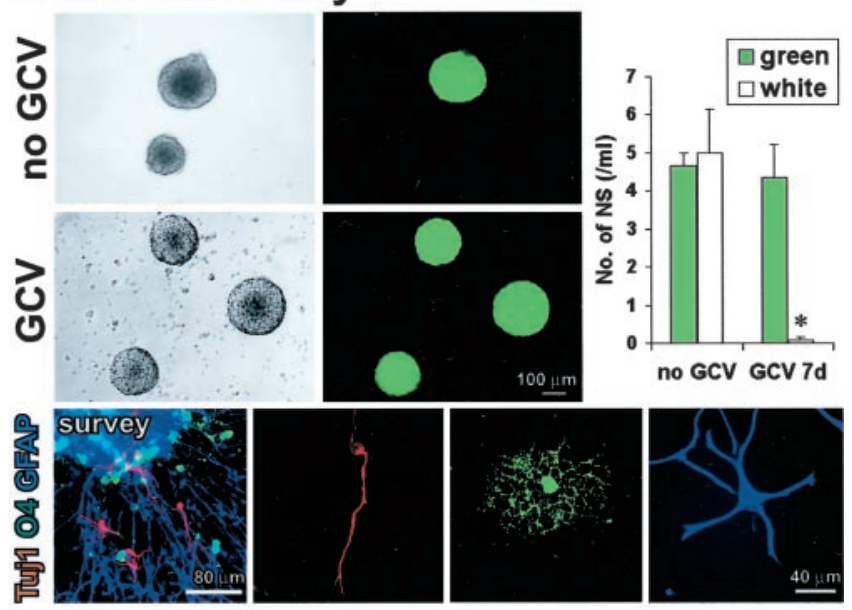

B. high density

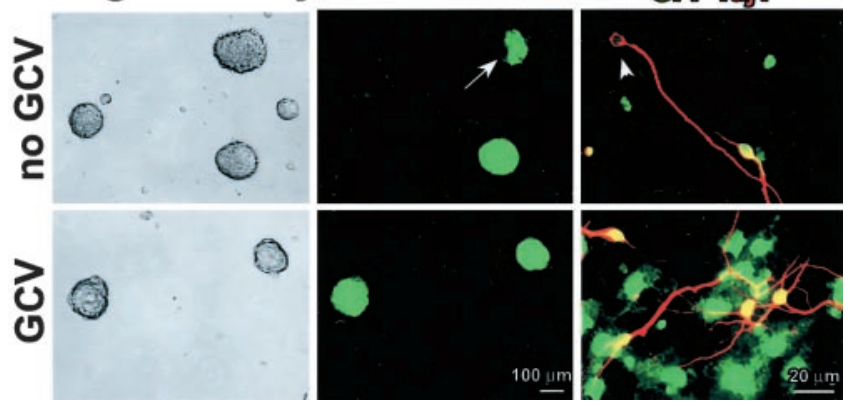

Figure 5. GFAP-expressing cells are not merely support cells, and their ablation is not nonspecifically toxic to neurosphere formation. A, Phase-contrast and fluorescent images of the same live floating neurospheres prepared at clonal cell density ( 1000 cells $/ \mathrm{ml} ; 500$ green cells plus 500 white cells) from primary P1 astrocyte cultures with mixed single-cell suspensions of GFP and GFAP-TK cells. Without GCV, both GFP-only (green) and GFAP-TK-only (white) spheres are present. There are no mixed GFP plus GFAP-TK (green and white) spheres. In the presence of GCV, only GFP (green) spheres are present. Graph shows mean \pm SEM number of green or white neurospheres (NS) formed per 1000 cells in the presence or absence of GCV. $n=3$ separate cultures prepared from different mice for each value. *Significantly different from non-GCVtreated or non-TK-expressing mice ( $p<0.01$; ANOVA plus post hoc pairwise analysis). Tricolored immunofluorescence of markers for neurons (Tuj1, red), oligodendrocytes (04, green), and astrocytes (GFAP, blue) shows that on differentiation, clonal spheres gave rise to different cells expressing markers of all three neural cell types, as depicted in a triple-labeled survey and details of individual cells of each type. $B$, Phase-contrast and fluorescent images of the same live floating neurospheres prepared at high cell density $(40,000 \mathrm{cell} / \mathrm{s} / \mathrm{ml})$ from primary $\mathrm{P} 1$ astrocyte cultures with mixed suspensions of GFP and GFAP-TK cells. In the absence of GCV, three types of spheres formed: GFP only (green), GFAP-TK only (white), and mixed GFP and GFAP-TK (green and white; arrow). In the presence of GCV, only GFP-only (green) spheres formed. Merged double-labeled images of immunocytochemistry for Tuj1 (red) plus GFP (green) show that differentiation of spheres grown without GCV gave rise to both GFP-positive (red plus green = yellow) and GFP-negative (red, arrowhead), Tuj1-positive neurons, whereas after differentiation of spheres grown with GCV, all Tuj1-positive neurons were also GFP positive (red plus green $=$ yellow).

most of these clonally derived spheres gave rise to all three types of neural cells (Fig. 5A). Clonal spheres derived from primary astrocyte cultures could be passaged to generate secondary spheres, which demonstrates their capacity for self-renewal. By comparing the starting cell density $(1000$ cells $/ \mathrm{ml})$ with the number of spheres generated (average 10 spheres $/ \mathrm{ml}$ ), we estimated that $\sim 1 \%$ of cells in the primary astrocyte cultures formed neurospheres. GCV treatment completely ( $>98 \%$ ) abolished the formation of clonal GFAP-TK-expressing spheres but did not re- 
duce the number of GFAP-TK-negative (i.e., GFP-expressing) spheres (Fig. 5A). These findings support the idea that the predominant NSC in primary astrocyte cultures is a GFAPexpressing cell and eliminate the possibility that the GCVinduced death of GFAP-TK-expressing cells that are not NSCs releases diffusible substances toxic to GFAP-negative NSCs; otherwise GFP-expressing spheres would not have survived. Nevertheless, clonal conditions could allow a potential non-GFAPexpressing NSC present in GFAP-TK cultures to initiate a neurosphere and grow in the presence of GCV at least until the phase of production of daughter GFAP-expressing cells. At this point, the GCV-induced death of dividing GFAP-TK cells might prevent additional maturation of the sphere either through loss of support functions (Song et al., 2002) or through a local toxic mechanism.

We next used chimeric neurosphere cultures prepared at high cell density to test whether differentiated GFAP-expressing astrocytes provide essential support for non-GFAP-expressing NSCs. Cell suspensions were prepared at a starting cell density of 40,000 cells/ml derived from equal numbers of GFP and GFAP-TK astroglia. Neurosphere cultures prepared from these suspensions yielded a mixture of approximately equal numbers of entirely GFP-positive spheres, entirely GFP-negative spheres, and chimeric spheres that were both GFP-positive and GFP-negative. On differentiation, chimeric spheres gave rise to both GFP-positive/ Tuj1-positive and GFP-negative/Tuj1-positive neurons in approximately equal numbers (Fig. 5B). GCV treatment abolished the formation of GFP-negative (white only) and chimeric spheres and yielded only spheres that were entirely GFP expressing (green only). On differentiation, only GFP-positive/Tuj1-positive neurons were present (Fig. 5B). The absence of chimeric (green and white) spheres and GFP-negative/Tuj1-positive neurons after GCV treatment demonstrates that neither contact-mediated nor diffusible support provided by TK-negative astrocytes derived from GFP mice was able to sustain the growth of any potential non-GFAP-expressing NSCs that might have been present.

To further test whether ablation of GFAP-expressing cells prevented the onset of neurosphere formation rather than neurosphere maturation, GCV was applied to confluent GFAP-TKexpressing astrocyte cultures for $24 \mathrm{hr}$ (GCV pulse), followed by thorough washout of GCV and passage into either primary astrocyte or sphere-forming conditions. A $1 \mathrm{~d}$ GCV pulse was not detectably toxic to confluent, nondividing GFAP-TK-expressing astroglia, and there was no apparent reduction in cell number (Fig. 6). Nevertheless, under these conditions, GCV will be phosphorylated by HSV-TK and remain trapped within the quiescent, transgene-expressing cells. We reasoned that this trapped, phosphorylated GCV should be able to kill transgene-expressing cells if they are then stimulated to divide. In agreement with this conjecture, a $24 \mathrm{hr}$ GCV pulse given to confluent GFAP-TKexpressing astrocytes killed all astrocytes when cell division was induced by passage under astrocyte conditions (Fig. 6). A $24 \mathrm{hr}$ GCV pulse to confluent GFAP-TK-expressing astrocytes similarly abolished ( $>98 \%$ reduction) the ability to form neurospheres from these cells (Fig. 6). These findings demonstrate that even in the absence of extracellular GCV, the phosphorylated GCV trapped inside individual quiescent GFAP-TK-expressing cells is sufficient to kill these cells after the induction of cell division. The failure to form neurospheres under these conditions argues strongly that the sphere-initiating cell expresses the GFAP-TK transgene and rules out the possibility that the failure of neurosphere formation is merely a result of the killing of

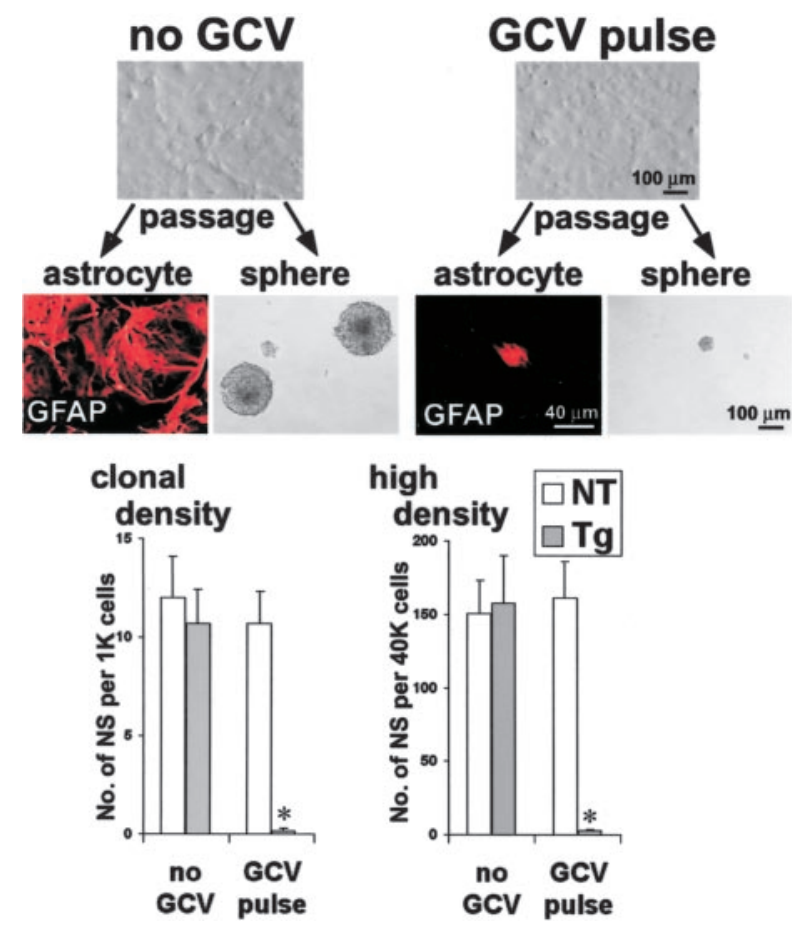

Figure 6. Ablation of GFAP-expressing cells prevents the onset of neurosphere formation. Confluent, nondividing primary P1 astrocyte cultures prepared from GFAP-TK transgenic mice were exposed to $\mathrm{GCV}$ for $24 \mathrm{hr}$ before passage (GCV pulse), with no detectable cell loss. Cultures not exposed to GCV could be passaged readily to form either more primary astrocytes or multipotent neurospheres. Cultures exposed to GCV failed to form either primary astrocytes or neurospheres after passage and the induction of cell division. Graphs show mean \pm SEM number of neurospheres (NS) prepared after passage of primary astrocyte cultures from nontransgenic (NT) or GFAP-TK transgenic $(T g)$ mice in the presence or absence of GCV pulse under clonal (1000 cells $/ \mathrm{ml})$ or high-density $(40,000$ cells $/ \mathrm{ml})$ conditions. The GCV pulse had no significant effect on the number of spheres formed by nontransgenic cells at either clonal or high density but abolished sphere formation by transgenic cells. $n=3$ separate cultures prepared from different mice. *Significantly different from non-GCV-treated or nontransgenic mice $(p<0.01$; ANOVA plus post hoc pairwise analysis).

GFAP-TK-expressing progeny of NSCs late in sphere development, either through loss of support or locally toxic mechanisms.

These findings demonstrate that (1) GFAP-expressing cells are not merely essential support cells for non-GFAP-expressing NSCs during neurosphere development and (2) failure of neurosphere formation in the present experiments was not attributable to a nonspecific toxic effect secondary to ablation of GFAPexpressing cells that are not NSCs. Together, the present findings thus far demonstrate that a GFAP-expressing cell is both necessary and sufficient for multipotent neurogenesis and that GFAPexpressing cells are the predominant NSCs that can be isolated from postnatal GZs.

\section{The predominant NSCs derived from adult GZs express GFAP in vitro and in vivo}

We next determined the relative contribution of GFAPexpressing cells to NSCs isolated from adult GZ tissue by examining the effect of GCV application in vitro on the ability to grow multipotent neurospheres from GZ tissue. In the absence of GCV, the number of neurospheres generated and the ability of these spheres to differentiate into all three types of neural cells did not differ between GZs from nontransgenic and transgenic adult mice. GCV added for the first 7 DIV had no detectable effect on sphere formation from nontransgenic mice but completely 
(>98\%) prevented neurosphere formation from GZ tissue derived from transgenic adult mice (Fig. $7 B, C$ ). GCV also prevented sphere formation when added only during the first $24 \mathrm{hr}$ in neurosphere cultures (Fig. 7C). GCV prevented the formation of secondary spheres after passage of primary neurospheres, thus eliminating the capacity for self-renewal.

We then characterized the overlap of GFAP and HSV-TK expression specifically in GZ cells in adult transgenic mice in vivo by conducting single-cell analysis using scanning confocal laser microscopy of periventricular forebrain tissue sections stained by immunohistochemistry for GFAP and TK. This analysis showed that GFAP-positive profiles identifiable as cells in periventricular GZs expressed transgene-derived TK, and that TK was present only in GFAP-expressing cells (Fig. 7A), in agreement with our previous analysis in other forebrain sites (Bush et al., 1999). Many GFAP-TK cells in the GZ exhibited a polarized morphology with a single prominent process (Fig. 7A) or, in some cases, a bipolar appearance.

To further test the contribution of GFAP-expressing cells to the neurogenic potential of adult GZs, we administered E-GCV to adult mice in vivo. E-GCV is a potent, lipophilic ester of GCV (Balzarini et al., 1998) that will cross the blood-brain barrier efficiently. Nontransgenic and transgenic mice were given $4 \mathrm{~d}$ of single daily intraperitoneal injections of E-GCV at $100 \mathrm{mg} \cdot \mathrm{kg}^{-1} \cdot \mathrm{d}^{-1}$. This in vivo experiment was based on logic similar to that used in the experiment described above, where a $24 \mathrm{hr}$ pulse of GCV delivered to quiescent transgene-expressing astroglia in vitro was sufficient to cause cell ablation after the cells were stimulated to divide (Fig. 6). In a similar manner, we reasoned that during $4 \mathrm{~d}$ of E-GCV delivery in vivo, GCV would be phosphorylated by all cells expressing the GFAP-TK transgene and would remain trapped within quiescent transgene-expressing cells. This trapped, phosphorylated GCV should then be able to kill any transgeneexpressing NSCs when they were stimulated to divide by placing them into neurosphere-forming conditions in vitro. In agreement with this conjecture, we found that $4 \mathrm{~d}$ of E-GCV given to adult transgenic mice in vivo largely abolished ( $>90 \%$ reduction) the ability to form neurospheres from GZ tissue, although no additional GCV was administered in vitro (Fig. $7 B, C$ ). E-GCV did not reduce neurosphere formation from nontransgenic mice (Fig. 7C). Because experimental evidence has shown that the doubling time of many NSCs is $>4$ d (Morshead et al., 1998; Doetsch et al., 1999), the finding that $4 \mathrm{~d}$ of E-GCV abolished the ability to derive neurospheres from adult GZs indicates that GFAP-TK must be expressed even by nondividing NSCs in vivo, and that these quiescent cells phosphorylated and trapped sufficient EGCV to be lethal when the cells divided after being placed into neurosphere-forming conditions in vitro. Together, these findings demonstrate that the predominant multipotent neurosphere-initiating
B
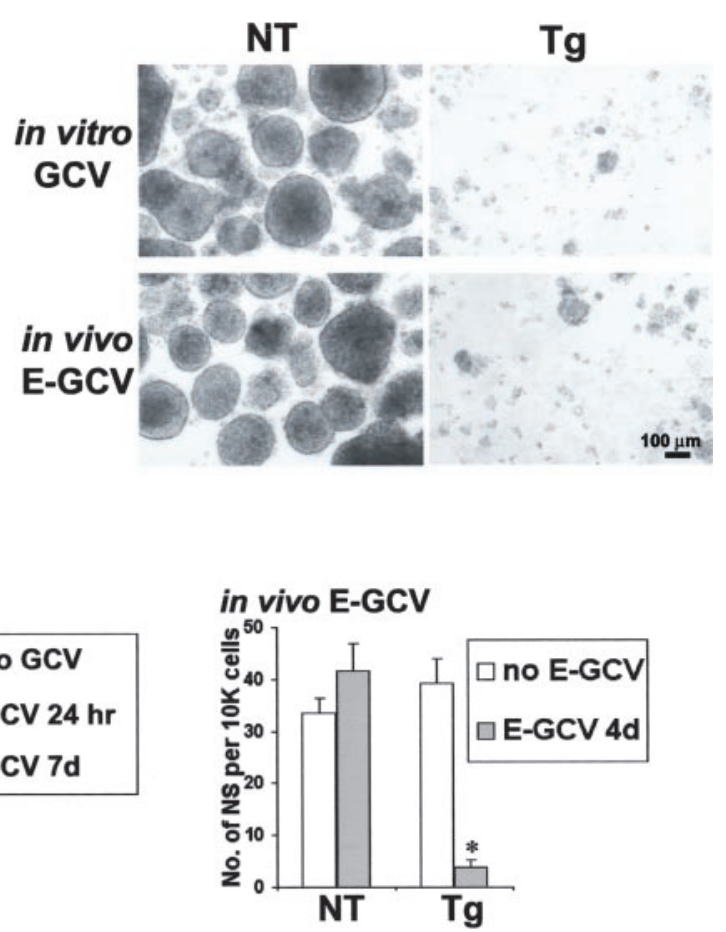

Figure 7. Ablation of GFAP-expressing cells by delivery of GCV in vitro or in vivo abolishes the ability to derive NSCs from adult GZ. A, Tissue section of GZ adjacent to lateral ventricle (LV) from an adult GFAP-TK transgenic mouse, double stained by immu( 政 nontransgenic mice. $n=3-5$ separate cultures prepared from different mice. *Significantly different from nontransgenic or non-GCV-treated mice ( $p<0.001$; ANOVA plus post hoc pairwise analysis).

cells and putative NSCs isolated from adult GZ, including quiescent NSCs, express GFAP both in vitro and in vivo.

\section{Discussion}

In this study, we used a transgenically targeted cell-ablation strategy in combination with quantitative evaluation of the ability to generate multipotent neurospheres to demonstrate that the predominant NSCs that are isolated from early postnatal and adult GZ express GFAP. In contrast, NSCs isolated from early embryonic GZ did not express GFAP. These findings are important with regard to the implications of GFAP expression, the potential for NSCs to change molecular phenotype during development, and the identity and origin of adult NSCs.

\section{Specificity of transgenically targeted ablation of dividing GFAP-expressing cells}

Transgenes insert randomly into the genome. For this reason, each line of transgenic mice is unique, and transgene expression can vary among different lines generated with the same promoter construct. Analysis of the extent of this variation for several transgene constructs showed that although variation from line to line was common, there was minimal variation among descendants of the same line (Feng et al., 2000). In addition, in most lines gen- 
erated, transgene expression occurred either in a majority or in restricted smaller subsets of cells that normally expressed the endogenous gene, whereas aberrant expression by other cell types was rare (Feng et al., 2000). Because of the considerable potential for variation, transgene expression must be characterized at the single-cell level for any line of transgenic mice studied, but once characterized, the expression within a given line is stable and is a useful tool (Feng et al., 2000).

The GFAP-TK transgenic mice used in the present study were generated with a maximal promoter cassette that contained the full sequence of the mouse GFAP gene (Johnson et al., 1995; Bush et al., 1998). Previous evaluation at the single-cell level of these mice showed that $98 \%$ of GFAP-expressing cells also expressed TK in the injured forebrain, and that all cells expressing detectable levels of TK also expressed GFAP (Bush et al., 1999). Here, we found that expression of transgene-derived TK reflects expression of the endogenous GFAP gene in forebrain periventricular GZ cells both in vivo and in vitro in these transgenic mice. Thus, cellular ablation in response to treatment with the antiviral agent GCV in these mice can be regarded as indicative of both the expression of GFAP and the occurrence of cell division.

\section{Implications of GFAP expression}

GFAP is an intermediate filament protein, as are nestin and vimentin (Lendahl et al., 1990; Eliasson et al., 1999; Eng et al., 2000). The expression of nestin and vimentin by NSCs or neural progenitors is well accepted, but for GFAP, such an association is potentially provocative. GFAP has been viewed historically as a marker for differentiated astrocytes. Nevertheless, various cell types inside and outside the CNS express GFAP, including cells in the liver, gut, kidney, lung, and other tissues, during development and in adults (Bush et al., 1998; Eng et al., 2000), and GFAP cannot be regarded as an exclusive astrocyte marker. A simple explanation compatible with the present data and those of others is that GFAP is merely one of several intermediate filaments, including nestin and vimentin, that are regulated dynamically and differentially in NSCs during different stages of maturation. Although one interpretation of the expression of GFAP by postnatal and adult NSCs could be that these cells are differentiated astrocytes, adult CNS tissue that contains many astrocytes but is distant from GZ does not exhibit NSC potential under standard conditions in vitro (Reynolds and Weiss, 1992; Morshead et al., 1994; Laywell et al., 2000; Seaberg and van der Kooy, 2002). The relationship between GFAP-expressing NSCs, GFAP-expressing astrocytes, and GFAP-expressing radial glia (see below) requires additional study, and it will be of interest to determine whether differentiated astrocytes have the potential to undergo reprogramming into NSCs or neural progenitors if stimulated appropriately (Kondo and Raff, 2000).

\section{Cells with NSC potential in vitro acquire GFAP expression during development}

The present findings indicate that cells with NSC potential in vitro are heterogeneous with regard to GFAP expression during development. NSCs derived at E12.5 did not express GFAP, whereas $\sim 50 \%$ of NSCs derived at E15.5 expressed GFAP, and essentially all postnatal and adult NSCs expressed GFAP, which suggests that during development, the predominant population of cells with NSC potential in vitro gradually acquires GFAP expression. These findings are compatible with reports that approximately one-half of the neural progenitor pool in E14 mouse cortex expresses a GFAP-regulated reporter gene (Heins et al., 2002), and that the onset of endogenous GFAP mRNA expression during mouse embryonic development occurs at this time (Andrae et al., 2001). These observations also suggest that cells with NSC potential in vitro may be present in multiple lineages or that a single lineage undergoes a change in phenotype from GFAP nonexpressing to GFAP expressing. These findings are compatible with and support other results showing the expression of GFAP by neuronal progenitor cells in postnatal and adult CNS in vivo (see below). Nevertheless, because the degree to which neurogenic potential in vitro reflects participation in neurogenesis in vivo is uncertain, additional studies will be required to determine whether GFAP-expressing cells contribute to neurogenesis during development in vivo.

\section{GFAP expression by adult NSCs}

The cellular identity of adult NSCs has been controversial. A claim that ependymal cells include a population of NSCs (Johansson et al., 1999) has not been confirmed by various in vivo and in vitro investigations (Chiasson et al., 1999; Laywell et al., 2000; Capela and Temple, 2002). The proposal that adult NSCs express GFAP and share ultrastructural characteristics with astroglia (Doetsch et al., 1999) is finding increasing experimental support, including the findings presented here. Previous studies have used single-cell lineage analysis to show that at least a portion of NSCs in late postnatal and adult GZ express GFAP in vivo and in vitro (Doetsch et al., 1999; Laywell et al., 2000). Other findings show that cells expressing a GFAP-GFP transgene during midembryonic and late embryonic development are NSCs and have characteristics of radial glia (Malatesta et al., 2000; Heins et al., 2002). At least a portion of NSCs partially purified by cell sorting for Lewis X also express GFAP (Capela and Temple, 2002). Our findings support and extend these observations by showing that the predominant population of NSCs that are derived from adult GZs express GFAP.

We identified NSCs as cells able to form multipotent neurospheres in vitro. Although the exact relationship between neurosphere-forming cells in vitro and NSCs in vivo is not understood, the ability to form multipotent neurospheres is the best current in vitro assay for the presence of putative NSCs. We showed that ablation of GFAP-expressing cells in vitro abolished the ability to derive multipotent neurospheres from postnatal and adult GZs. Control experiments, including clonal analysis, demonstrated that failure of neurosphere growth was not merely secondary to loss of GFAP-expressing support cells, nor was it caused by a nonspecific toxic effect. We also showed that $4 \mathrm{~d}$ of E-GCV given to adult transgenic mice in vivo abolished the ability to form neurospheres from adult GZ tissue. NSC doubling time is thought to be $>6 \mathrm{~d}$ and probably $>15 \mathrm{~d}$ (Morshead et al., 1998; Doetsch et al., 1999). Thus, over a $4 \mathrm{~d}$ period, only a portion of NSCs will undergo cell division and be killed in vivo by E-GCV treatment. The finding that $4 \mathrm{~d}$ of E-GCV in vivo abolished the ability to derive neurospheres indicates that GFAP-TK must be expressed by quiescent NSCs, and that these cells phosphorylated and trapped sufficient E-GCV to be killed when they attempted to divide after being placed into neurosphere-forming conditions in vitro. We conclude that the predominant population of cells with NSC potential in the adult periventricular area in vivo, including quiescent NSCs, express GFAP and GFAP-TK.

It is unclear why NSCs express various intermediate filament proteins, including GFAP, and differentially regulate their expression during development. The deletion of GFAP expression by targeted gene knock-out does not appear overtly to disturb brain development or to perturb either neurogenesis or gliogenesis (Gomi et al., 1995; Pekny et al., 1995), which suggests that the 
roles played by GFAP in NSCs are not unique and that there is a capacity for redundancy of function among different intermediate filament proteins. Nevertheless, although GFAP expression may not be essential for NSC function, the expression of GFAP by postnatal and adult NSCs in vivo and in vitro provides an additional means of investigating their cellular identity, developmental origins, and cell biology by transgenic and other techniques. It is also interesting to consider that the expression of GFAP by late embryonic, postnatal, and adult NSCs may reflect their relationship to radial glia. Radial glia have been shown to be NSCs during late embryonic development (Malatesta et al., 2000; Noctor et al., 2001; Heins et al., 2002), and radial glia adopt GFAP expression as development progresses (Levitt and Rakic, 1980; Voigt, 1989; Mission et al., 1991). GFAP-expressing NSCs that are present in adult GZ may thus derive directly from radial glia or may simply be radial glia that express GFAP and persist past development.

\section{References}

Alvarez-Buylla A, Garcia-Verdugo JM, Tramontin AD (2001) A unified hypothesis on the lineage of neural stem cells. Nat Rev Neurosci 2:287-293.

Anderson DJ (2001) Stem cells and pattern formation in the nervous system: the possible versus the actual. Neuron 30:19-35.

Andrae J, Bongcam-Rudloff E, Hansson I, Lendahl U, Westermark B, Nister M (2001) A $1.8 \mathrm{~kb}$ GFAP-promoter fragment is active in specific regions of the embryonic CNS. Mech Dev 107:181-185.

Balzarini J, Degreve B, Andrei G, Neyts J, Sandvold M, Myhren F, de Clercq E (1998) Superior cytostatic activity of the ganciclovir elaidic acid ester due to the prolonged intracellular retention of ganciclovir anabolites in herpes simplex virus type 1 thymidine kinase gene-transfected tumor cells. Gene Ther 5:419-426.

Bush TG, Savidge TC, Freeman TC, Cox HJ, Campbell EA, Mucke L, Johnson MH, Sofroniew MV (1998) Fulminant jejuno-ileitis following ablation of enteric glia in adult transgenic mice. Cell 93:189-201.

Bush TG, Puvanachandra N, Horner CH, Polito A, Ostenfeld T, Svendsen CN, Mucke L, Johnson MH, Sofroniew MV (1999) Leukocyte infiltration, neuronal degeneration, and neurite outgrowth after ablation of scarforming, reactive astrocytes in adult transgenic mice. Neuron 23:297-308.

Capela A, Temple S (2002) LeX/ssea-1 is expressed by adult mouse CNS stem cells, identifying them as nonependymal. Neuron 35:865-875.

Chiasson BJ, Tropepe V, Morshead CM, van der Kooy D (1999) Adult mammalian forebrain ependymal and subependymal cells demonstrate proliferative potential, but only subependymal cells have neural stem cell characteristics. J Neurosci 19:4462-4471.

Davis AA, Temple S (1994) A self-renewing multipotential stem cell in embryonic rat cerebral cortex. Nature 372:263-266.

Doetsch F, Caille I, Lim DA, Garcia-Verdugo JM, Alvarez-Buylla A (1999) Subventricular zone astrocytes are neural stem cells in the adult mammalian brain. Cell 97:703-716.

Eliasson C, Sahlgren C, Berthold CH, Stakeberg J, Celis JE, Betsholtz C, Eriksson JE, Pekny M (1999) Intermediate filament protein partnership in astrocytes. J Biol Chem 274:23996-23406.

Eng LF, Ghirnikar RS, Lee YL (2000) Glial fibrillary acidic protein: GFAPthirty-one years (1969-2000). Neurochem Res 25:1439-1451.

Feng G, Mellor R, Bernstein M, Keller-Peck C, Nguyen Q, Wallace M, Nerbonne J, Lichtman J, Sanes J (2000) Imaging neuronal subsets in transgenic mice expressing multiple spectral variants of GFP. Neuron 28:41-51.

Gage FH (2000) Mammalian neural stem cells. Science 287:1433-1438.

Geschwind DH, Ou J, Easterday MC, Dougherty JD, Jackson RL, Chen Z, Antoine H, Terskikh A, Weissman IL, Nelson SF, Kornblum HI (2001) A genetic analysis of neural progenitor differentiation. Neuron 29:325-339.

Gomi H, Yokoyama T, Fujimoto K, Ikeda T, Katoh A, Itoh T, Itohara S (1995) Mice devoid of the glial fibrillary acidic protein develop normally and are susceptible to scrapie prions. Neuron 14:29-41.

Groszer M, Erickson R, Scripture-Adams DD, Lesche R, Trumpp A, Zack JA, Kornblum HI, Liu X, Wu H (2001) Negative regulation of neural stem/ progenitor cell proliferation by the Pten tumor suppressor gene in vivo. Science 294:2186-2189.

Heins N, Malatesta P, Cecconi F, Nakafuku M, Tucker KL, Hack MA,
Chapouton P, Barde YA, Götz M (2002) Glial cells generate neurons: the role of the transcription factor Pax6. Nat Neurosci 5:308-315.

Johansson CB, Momma S, Clarke DL, Risling M, Lendahl U, Frisen J (1999) Identification of a neural stem cell in the adult mammalian central nervous system. Cell 96:25-34.

Johnson WB, Ruppe MD, Rockenstein EM, Price J, Sarthy VP, Vederber LC, Mucke L (1995) Indicator expression directed by regulatory sequences of the glial fibrillary acidic protein (GFAP) gene: in vitro comparison of distinct GFAP-lacZ transgenes. Glia 13:174-184.

Kondo T, Raff M (2000) Oligodendrocyte precursor cells reprogrammed to become multipotential CNS stem cell. Science 289:1754-1757.

Laywell ED, Rakic P, Kukekov VG, Holland EC, Steindler DA (2000) Identification of a multipotent astrocytic stem cell in the immature and adult mouse brain. Proc Natl Acad Sci USA 97:13883-13888.

Lendahl U, Zimmerman LB, McKay RD (1990) CNS stem cells express a new class of intermediate filament protein. Cell 60:585-595.

Levitt P, Rakic P (1980) Immunoperoxidase localization of glial fibrillary acidic protein in radial glial cells and astrocytes of the developing rhesus monkey brain. J Comp Neurol 193:815-840.

Luskin MB (1993) Restricted proliferation and migration of postnatally generated neurons derived from the forebrain subventricular zone. Neuron 11:173-189.

Malatesta P, Hartfuss E, Götz M (2000) Isolation of radial glial cells by fluorescent-activated cell sorting reveals a neuronal lineage. Development 127:5253-5263.

Mission JP, Takahashi T, Caviness Jr VS (1991) Ontogeny of radial and other astroglial cells in murine cerebral cortex. Glia 4:138-148.

Morshead CM, Reynolds BA, Craig CG, McBurney MW, Staines WA, Morassutti D, Weiss S, van der Kooy D (1994) Neural stem cells in the adult mammalian forebrain: a relatively quiescent subpopulation of subependymal cells. Neuron 13:1071-1082.

Morshead CM, Craig CG, van der Kooy D (1998) In vivo clonal analyses reveal the properties of endogenous neural stem cell proliferation in the adult mammalian forebrain. Development 125:2251-2261.

Mucke L, Oldstone MBA, Morris JC, Nerenberg MI (1991) Rapid activation of astrocyte-specific expression of GFAP-lacZ transgene by focal injury. New Biol 3:465-474.

Noctor SC, Flint AC, Weissman TA, Dammerman RS, Kriegstein AR (2001) Neurons derived from radial glial cells establish radial units in neocortex. Nature 409:714-720.

Okabe M, Ikawa M, Kominami K, Nakanishi T, Nishimune Y (1997) “Green mice" as a source of ubiquitous green cells. FEBS Lett 407:313-319.

Pekny M, Leveen P, Pekna M, Eliasson C, Berthold CH, Westermark B, Betsholtz C (1995) Mice lacking glial fibrillary acidic protein display astrocytes devoid of intermediate filaments but develop and reproduce normally. EMBO J 14:1590-1598.

Reynolds BA, Weiss S (1992) Generation of neurons and astrocytes from isolated cells of the adult mammalian central nervous system. Science 255:1707-1710.

Rietze RL, Valcanis H, Brooker GF, Thomas T, Voss AK, Bartlett PF (2001) Purification of a pluripotent neural stem cell from the adult mouse brain. Nature 412:736-739.

Seaberg RM, van der Kooy D (2002) Adult rodent neurogenic regions: the ventricular subependyma contains neural stem cells, but the dentate gyrus contains restricted progenitors. J Neurosci 22:1784-1793.

Song H, Stevens CF, Gage FH (2002) Astroglia induce neurogenesis from adult neural stem cells. Nature 417:39-44.

Suslov ON, Kukekov VG, Ignatova TN, Steindler DA (2002) Neural stem cell heterogeneity demonstrated by molecular phenotyping of clonal neurospheres. Proc Natl Acad Sci USA 99:14506-14511.

Svendsen CN, Bhattacharyya A, Tai YT (2001) Neurons from stem cells: preventing an identity crisis. Nat Rev Neurosci 2:831-834.

Temple S (2001) The development of neural stem cells. Nature 414:112-117.

Tropepe V, Sibilia M, Ciruna BG, Rossant J, Wagner EF, van der Kooy D (1999) Distinct neural stem cells proliferate in response to EGF and fibroblast growth factor in the developing mouse telencephalon. Dev Biol 208:166-188.

Voigt T (1989) Development of glial cells in the cerebral wall of ferrets: direct tracing of their transformation from radial glia into astrocytes. J Comp Neurol 289:74-88. 